\title{
PRODUTOS VERDES: UM ESTUDO SOBRE ATITUDE, INTENÇÃO E COMPORTAMENTO DE COMPRA DE UNIVERSITÁRIOS BRASILEIROS PRODUTOS VERDES: UM ESTUDO SOBRE
ATITUDE, INTENÇÃO E COMPORTAMENTO DE
COMPRA DE UNIVERSITÁRIOS BRASILEIROS
}

Data de submissão: 19-12-2013 Aceite: 03-06-2015

Sofia Batista Ferraz ${ }^{1}$

Cláudia Buhamra Abreu Romero² Sílvia Maria Dias Pedro Rebouças ${ }^{3}$ Josimar Souza Costa ${ }^{4}$

\section{RESUMO}

Para conhecer melhor o consumidor e o processo de compra de produtos verdes, torna-se necessário não apenas identificar o perfil do consumidor ambientalmente responsável, mas também desenvolver e aplicar mensurações relevantes acerca do seu comportamento ecologicamente consciente. O objetivo desta pesquisa é analisar as relações e o possível gap entre atitude, intenção e comportamento de compra de produtos verdes entre universitários brasileiros da área de gestão. O estudo é de natureza quantitativa e é descritiva quanto aos seus objetivos. Trata-se de uma pesquisa de campo com a utilização da técnica de Modelagem em Equações Estruturais. Os resultados confirmaram que existe uma relação positiva entre atitude e intenção de compra de produtos verdes, assim como a há uma relação positiva entre intenção e comportamento, apesar de menos expressiva. A influência da disponibilidade, preço e qualidade percebidos entre intenção e comportamento de compra também foi comprovada. Apesar das diferenças de influência dos construtos, notou-se que o preço percebido é uma variável preponderante durante a transição entre intenção e comportamento de compra de produtos verdes. Espera-se contribuir para a geração de debates acerca da aquisição de produtos verdes, estimular o desenvolvimento de novas pesquisas, além de auxiliar na elaboração de estratégias de Marketing baseadas no comportamento de seus consumidores.

Palavras-chave: Consumo verde, Atitude, Intenção, Comportamento de compra.

\footnotetext{
1 Possui Graduação em Publicidade e Propaganda pela Universidade de Fortaleza, UNIFOR. Graduação em Administração pela Universidade Federal do Ceará, UFC. Mestrado em Administração e Controladoria pela Universidade Federal do Ceará, UFC. Doutorado em andamento em Administração Universidade de São Paulo, USP. São Paulo. São Paulo. Brasil. E-mail: sofiasbf@gmail.com

2 Possui Graduação em Administração de Empresas pela Universidade Federal do Ceará. Mestrado em Administração de Empresas pela Fundação Getúlio Vargas - São Paulo, FGV - SP. Doutorado em Administração de Empresas pela Fundação Getúlio Vargas - São Paulo, FGV -SP. Fortaleza. Ceará. Brasil. E-mail: buhamra@ufc.br

3 Possui Graduação em Matemática Aplicada e Computação pelo Instituto Superior Técnico da UTL, IST, Portugal. Mestrado em Gestão Empresarial pela Faculdade de Economia da Universidade do Algarve, FE-UALG, Portugal. Doutorado em Estatística e Investigação Operacional pela Faculdade de Ciências da Universidade de Lisboa, FCUL, Portugal. Atualmente está em Pós-Doutorado na Universidade de Salamanca e na Universidade do Algarve. Professora adjunta na Faculdade de Economia, Administração, Atuária e Contabilidade (FEAAC) da Universidade Federal do Ceará (UFC) e docente permanente do Programa de Pós-Graduação em Administração e Controladoria da UFC. Fortaleza. Ceará. Brasil. E-mail: smdpedro@gmail.com

4 Possui Curso técnico/profissionalizante pelo Instituto Federal do Ceará - Reitoria, IFCE. Graduação em Administração pela Universidade de Fortaleza, UNIFOR. Mestrado em Administração e Controladoria pela Universidade Federal do Ceará, UFC. Doutorado em andamento em Administração de Empresas pela Universidade de Fortaleza, UNIFOR. Fortaleza. Ceará. Brasil. E-mail: josimarscosta@gmail.com
} 


\section{ABSTRACT}

In order to learn more about consumer and the process of buying green products, it becomes necessary not only to identify the profile of environmentally responsible consumer, but also to develop and apply relevant metrics about your environmentally conscious behavior. This research aims to analyze the relationships and the possible gap between attitude and behavior intention to purchase green products among Brazilian university management area. The study is quantitative in nature and is descriptive of your goals. This is a field study using the technique of Structural Equation Modeling. The results confirmed the positive relationship between attitude and intention to buy green products, as well as the positive relationship between intention and behavior, although less expressive. The influence of availability, perceived price and quality between intention and purchase behavior was also confirmed. Despite differences in the influence of the constructs, was noted that the price realized is a predominant variable during the transition between intention and purchase behavior of green products. Expected to contribute to the generation of discussions about procurement of green products, stimulate the development of new research, and assist in developing marketing strategies based on the behavior of their consumers.

Keywords: Green consumption, Attitude, Intention, Purchase behavior.

\section{INTRODUÇÃO}

Com o desenvolvimento da economia na segunda metade do século XX, que acarretou um aumento de até quatro vezes na produção de manufaturas entre 1950 e 1970 e um crescimento sem precedentes na comercialização dos produtos manufaturados (HOBSBAWN, 1994; GOLDEMBERG; VILLANUEVA, 2003), os modelos de interação do ser humano com o meio ambiente após a Revolução Industrial eram demarcados essencialmente pela busca do lucro e ganhos socioeconômicos (ALMEIDA JUNIOR, 1994). Tais fatos, conjugados aos hábitos de consumo das sociedades contemporâneas e à constante manutenção do padrão de vida das pessoas, desencadearam uma série de consequências ao meio ambiente, como a poluição, as queimadas do solo, a extinção da biodiversidade, o aumento da produção de lixo, as mudanças climáticas e o desperdício de água e alimentos.

Décadas depois, devido à paulatina popularização e à consolidação da preocupação ambiental, conforme esclarece a Confederation of British Industry (CBI, 2007), tornou-se possível identificar os três co-protagonistas responsáveis pela mudança de paradigmas relacionados ao meio ambiente: o governo, com a incumbência de desenvolver um ambiente propício a mudanças e acordos com demais países; os consumidores, que podem ser considerados propulsores com suporte nos seus comportamentos de compra, e o setor empresarial, que tem o poder e o dever de investir e fazer com que as causas sustentáveis se tornem realidade.

Quanto a este último, Webster Jr. (1975) informa que estimular e servir aos interesses públicos quanto às questões ambientais deve ser uma prioridade para as organizações. Além de acarretar benefícios concretos para a comunidade, Ottman (1993) entende que o "esverdeamento" da organização configura-se como um atributo de diferenciação diante do mercado competitivo vivenciado diariamente pelas empresas. A utilização do conceito ambiental em estratégias organizacionais como vantagem estratégica é bastante debatida no âmbito acadêmico (GHAHREMANI, 1990; MAIMON, 1994; DONAIRE, 1996; MENON; MENON, 1997; POLONSKY; ROSEMBERGER III, 1995). Essa vantagem deve-se, principalmente, em resposta ao surgimento do perfil de consumidor que se importa, cada vez mais, com o amadurecimento de uma consciência coletiva e do consumo cidadão (FRANZEN; MEYER, 2010; NASCIMENTO et al., 2014; SOUZA; CASOTTI, LEMME, 2013). Esse amadurecimento reflete-se na crescente oferta de produtos verdes que, de acordo com Ottman (1993), podem ser conceituados como produtos que impactam menos no 
meio ambiente do que seus concorrentes e demais alternativas. De forma complementar, são ainda aqueles que podem ser desenvolvidos baseados em normas ou selos ecológicos que satisfaçam à demanda do consumidor a partir de ações como a utilização de materiais reciclados/ recicláveis e redução de embalagens ou recursos naturais raros (LAMBIN, 2002).

Para conhecer melhor o consumidor e o processo de compra de produtos verdes, tornase necessário não apenas identificar o perfil do consumidor ambientalmente responsável, mas também desenvolver e aplicar mensurações relevantes acerca do seu comportamento ecologicamente consciente (WEBSTER JR, 1975).

$O$ consumo de produtos verdes ainda firma os primeiros passos em muitos países emergentes (DERKSEN; GARTRELL, 1993; ALI; KHAN; AHMED, 2011): à medida que as sociedades atingem um estágio satisfatório de desenvolvimento e tornam-se mais influentes, os seus membros, antes consternados com embates econômicos que visassem a sobrevivência, hoje estão mais livres para buscar objetivos pós-materialistas, tais como a liberdade política e a proteção ambiental (FRANZEN; MEYER, 2010). A conscientização das pessoas acerca da importância do meio ambiente e da depleção de recursos naturais por parte do processo de produção e consumo configura, portanto, uma constante cultural no ocidente (SEROA DA MOTTA, 2002). Trata-se, conforme Guimarães (1992, p.38), da apropriação de "um novo paradigma de desenvolvimento que deve permitir uma profunda revisão das práticas atuais de incorporação do patrimônio natural, através de novas formas de organização social e de novos padrões de produção e consumo".

Com base nesse novo paradigma e amparado pela Teoria da Ação Racional (AJZEN; FISHBEIN, 1980; FISHBEIN; AJZEN, 1975), Chan (2001) sugere o modelo que demonstra a relação entre atitude, intenção e comportamento de compra de produtos verdes, representados por Green Purchase Attitude (GPA), Green Purchase Intention (GPI) e Green Purchase Behavior (GPB) respectivamente. A pesquisa, realizada em território chinês, buscou propor e verificar o modelo empírico, além de analisar possíveis antecedentes ao processo de compra de produtos verdes. O seu trabalho, depois de validado, foi replicado por Afonso (2010) em Portugal para estudar o perfil do consumidor verde, por Reyes-Ricon (2010) para estudar os antecedentes do consumo verde no Brasil e por Ali, Khan e Ahmed (2011) para verificar a influência de variáveis mediadoras no processo de compra de produtos verdes no Paquistão.

O modelo e as escalas propostas por Chan (2001) ainda não foram aplicados na realidade brasileira e, tampouco, entre estudantes da área de gestão. D'Souza, Taghian e Khosla (2007) acrescentam ainda ao modelo de Chan, que será utilizado nesta pesquisa, Situational Factors (SF) ou fatores situacionais compostos pela disponibilidade, qualidade e preço percebidos enquanto influenciadores da intenção e comportamento de compra de produtos verdes. Ademais, de acordo com estudo empírico empreendido por Leonidou e Leonidou (2011), noventa e cinco por cento (95\%) da literatura sobre marketing e gestão verde dos últimos 30 anos é proveniente de autores estadunidenses $(55,6 \%)$ e europeus $(39,4 \%)$, denotando uma lacuna existente para a aplicação em mercados emergentes.

É válido informar ainda que se sabe pouco acerca da visão de estudantes graduandos da área de negócios (CORDANO et al., 2010), que esses jovens possuem valores que influenciarão a gestão de futuros líderes e executivos no que concerne a preocupações e demandas ambientais (MARQUES, 1998; ROCHA; MARQUES, 2004) e, ainda, que o nível de educação é uma importante variável a ser relacionada com atitudes e comportamentos verdes (SCHWARTZ; MILLER, 1991; VAN LIERE; DUNLAP, 1981; DIAMANTOPOULOS et al., 2003). A partir destas conjunturas, a pergunta norteadora da pesquisa é "Qual é a relação entre a atitude, a intenção e o comportamento de compra de produtos verdes por parte dos consumidores universitários da área de gestão do Brasil?". 
Neste sentido, o objetivo do artigo é analisar as relações e o possível gap entre atitude, intenção e comportamento de compra de produtos verdes de universitários da área de gestão. Delineiam-se, consequentemente, os seguintes objetivos específicos: identificar a relação entre atitude e intenção de compra de produtos verdes (GPA e GPI, respectivamente); identificar a relação entre intenção e comportamento de compra de produtos verdes (GPI e GPB, respectivamente); e analisar as características do gap entre a intenção (GPI) e o comportamento de compra (GPB) de produtos verdes, considerados os fatores situacionais (SF) formados pela disponibilidade, preço e qualidade percebidos dos produtos.

\section{REFERENCIAL TEÓRICO}

\subsection{Atitude}

Dentre os conceitos-chave utilizados, pela área de conhecimento da Psicologia e da Mercadologia, no que concerne ao Comportamento do Consumidor, tais quais memória e cognição, afeto e emoção, julgamento e tomada de decisão e dinâmica de grupo, destaca-se o estudo das atitudes (AJZEN, 2008). Sua importância já era, desde cedo, ratificada por Aaker e Myers (1987, p. 160), quando argumentavam que "a atitude relativa a um produto ou marca é o pilar sobre o qual estão firmados as vendas e os lucros de grandes corporações". De maneira mais detalhada, as atitudes são boas preditoras de comportamento, como, por exemplo, no caso de uma pessoa ser favorável a determinada ideia ou partido político, tal fato pode determinar uma inclinação de voto em futuras eleições; acarretam, ainda, funções específicas, por serem organizadoras e compiladoras de informações que se tem acerca de determinado objeto; além de funcionarem também como base para situações sociais, com o uso dessa compilação de informações como forma de justificar o comportamento do sujeito (RODRIGUES, 1976). As atitudes configuram-se como crenças e sentimentos acerca de um objeto que predispõe o sujeito a se comportar de maneira consistente em relação a ele (FISHBEIN; AJZEN, 1975). Para Solomon (2011), atitude é uma avaliação duradoura das pessoas, sobre elas e acerca dos objetos e ambientes que as cercam. Rodrigues, Assmar e Jablonski (2003, p.100) complementam quando dizem que consiste em "uma organização duradoura de crenças e cognições em geral, dotada de carga afetiva pró ou contra um objeto social definido, que predispõe a uma ação coerente com as cognições e afetos relativos a este objeto". Dentre os pioneiros no estudo de atitudes, é relevante, também, abordar a definição de Thurstone (1976, p.158), para quem se trata da "[...] soma de inclinações e sentimentos, preconceitos ou distorções, noções pré-concebidas, ideias, temores, ameaças e convicções de um indivíduo acerca de qualquer assunto específico".

As pessoas possuem tendências em responder positiva ou negativamente a determinado estímulo ou objeto - podendo este ser uma ideia, alguém, um grupo ou uma situação. Desta forma, a pessoa fica predisposta a pensar e raciocinar de determinada maneira, o que acarreta a possível previsão de comportamento, seja relacionada ao consumo ou a qualquer aspecto da sua vida. Rodrigues (1976) assevera que o conhecimento e a previsão das atitudes permitem que realizem inferências sobre o comportamento. De acordo com Velter et al. (2009), os estudos das atitudes consistem em objetivo fundamental para entender a intenção do comportamento por tratar-se de um determinante primordial da mesma. Em suma, percebe-se que determinada crença a respeito de um objeto conduz à formação de uma atitude que, por sua vez, orienta a formação de uma série de intenções em relação ao comportamento acerca do objeto (FISHBEIN; AJZEN, 1975; CHAN, 2001; PEREIRA; AYROSA, 2004). Sugere-se, desta forma, a seguinte hipótese: 
H1 - GPA está positivamente relacionado ao GPI.

De acordo com Stern e Oskamp (1987), a mensuração das atitudes é também considerada relevante por permitir prever ações e comportamentos ambientalmente responsáveis, além de fornecer suporte às estratégias de Marketing das organizações.

Nota-se que o consumidor consciente tem atitudes diferenciadas que, de acordo com Dias (2008), podem ser delineadas com origem na consciência, posicionamento e atividades ecológicas. A primeira, relacionada à consciência, diz respeito às crenças e conhecimentos acerca da causa ecológicos, intimamente relacionados aos dados e informações recebidos e processados. O posicionamento relaciona-se à dimensão afetiva acerca dos produtos verdes, enquanto a atividade traduz-se como o fato de agir de modo ecologicamente correto.

As preocupações expressas pelas atitudes ambientais das pessoas influenciam positivamente o comportamento em face do meio ambiente (KINNEAR, TAYLOR, 1973; GOSKEN; ADAMAN; ZENGINOBUZ, 2002). Alwitt e Pitts (1996) exprimem que, no contexto ecológico, apesar de as atitudes em prol do meio ambiente serem consideradas pelos profissionais da área mercadológica, não desencadeará - necessariamente - no comportamento de compra do produto ecologicamente correto.

\subsection{Intenção de compra}

Prever qual será o comportamento das pessoas, na qualidade de consumidoras, é uma das melhores habilidades que uma organização pode possuir (BLACKWELL, MINIARD; ENGEL, 2005). As intenções podem ser introduzidas, portanto, de acordo com Malhotra e McCort (2001), como o plano consciente para alguém realizar um dado comportamento ou até um julgamento particular de como será o comportamento futuro, relativo à aquisição, disposição e utilização de produtos e serviços (BLACKWELL, MINIARD; ENGEL, 2005; MOWEN; MINOR, 2003). A intenção de realizar um comportamento é, constantemente, confundida com o construto de atitude. Tal confusão pode ser justificada no fato de que quanto mais forte uma atitude em relação a um objeto, maior será a intenção de realizar determinado comportamento referente a ele. Determinada por quatro elementos essenciais, como comportamento, objetivo, situação e ocasião, a intenção só pode ser acurada se houver a mesma medição para os demais componentes normativos e atitudinais (FISHBEIN; AJZEN, 1975).

Mensurações de intenção de compra têm sido frequentemente utilizadas para identificar nichos e potencialidades para os produtos (WHITLAR, GEURTS, SWENSON, 1993), pois, segundo Berkmam e Gilson (1979), quanto maior a intenção, maior é a probabilidade do comportamento de compra se concretizar.

Ainda que não possamos garantir que os consumidores vão agir de acordo com suas intenções, existem algumas coisas que podemos controlar ou pelo menos ter a consciência de que vão influenciar a acurácia previsiva das intenções. A medida das intenções é importante. As intenções medidas devem corresponder exatamente ao comportamento a ser previsto (BLACKWELL; MINIARD; ENGEL, 2005, p. 295).

A intenção foi introduzida como componente mediador entre atitudes e comportamento, isto é, as atitudes servem para nortear a intenção e esta, por sua vez, influenciar o comportamento - agindo como uma espécie de índice que uma pessoa estaria disposta a tentar desempenhar em prol de uma ação. Fishbein e Ajzen (1975), precursores em estudos sobre modelos de comportamento, informam ainda que o preditor imediatamente mais relevante ao comportamento é a sua intenção: apesar das possíveis diferenças que possam surgir entre intenção e 
comportamento, pesquisas empíricas já apontaram que estas variáveis registram altos índices de correlação. Com base nisso, é proposta a hipótese que segue:

H2 - GPI está positivamente relacionado ao GPB.

Um fator importante apontado por Fishben e Ajezen (1980) é que a correlação entre intenção e comportamento pode ser consolidada à medida que existe um curto intervalo de tempo entre a aferição e mensuração.

Deve-se salientar que todo o estudo acerca de intenções de comportamento não remete a respostas exatas. Intenções, assim como atitudes, podem mudar. Velter et al. (2009) afirmam ainda que a intenção do consumidor verde resulta do trade-off percebido entre as consequências ambientais e as consequências individuais da compra, o que pode mostrar o porquê de consumidores com alta consciência ecológica não efetivarem o comportamento de compra verde. De qualquer forma, geralmente, monitorar as intenções de consumidores e possíveis clientes contribui de modo eficaz para a elaboração de estratégias mercadológicas (BLACKWELL; MINIARD; ENGEL, 2005).

\subsection{Comportamento de compra}

Os consumidores, à medida que se desenvolvem inúmeras preocupações como a degradação ambiental e demais problemas, como a poluição e o aquecimento global, percebem que o seu comportamento de compra pode causar um elevado impacto para o meio ambiente e para a sociedade (WAHID; RAHBAR; SHYAN, 2011).

Jackson (2005) assume o argumento de que a escolha de comportamentos e estilos de vida constitui um papel vital para o alcance de um desenvolvimento sustentável. Tal afirmação, de acordo com o autor, é um dos pontos de concordância mais emergentes dos diversos debates realizados nas últimas décadas. A preocupação do consumidor acerca de questões ambientais pode não ser traduzida em comportamento ecologicamente correto; comportamento este que pode ser definido, de acordo com Corral-Verdugo e Armendariz (2000, p.471), como "o conjunto de ações dirigidas, deliberadas e efetivas que respondem a requerimentos sociais e individuais e que resultam na proteção do meio". De qualquer forma, portadores de fortes crenças de que seu comportamento irá acarretar em consequências positivas para a causa tendem a se engajar mais em comportamentos que sirvam de apoio ao meio ambiente.

Com suporte na importância desempenhada pelo entendimento do comportamento do consumidor, Fishbein e Ajzen (1975) e Cronin, Brady e Hult (2000) informam que a classe mais comum de modelos é o comportamento-intenção (behavioral-intention ou $\mathrm{BI}$ ), de modo a assumir que o comportamento acerca de determinado objeto é aproximado pela intenção de realizar tal comportamento (MALHOTRA; MCCORT, 2001).

De acordo com Mostafa (2006), o comportamento de compra de produtos verdes deve ser definido como o consumo de produtos benevolentes ou benéficos para o meio ambiente, recicláveis ou conserváveis, sensíveis e também responsáveis às preocupações ambientais. $\mathrm{O}$ autor também diferencia os conceitos de atitude e comportamento. Enquanto atitude remete ao grau de adesão a valores e opiniões acerca das empresas e dos consumidores relacionados a temáticas como sustentabilidade e responsabilidade social, comportamento diz respeito à prática, no dia a dia, de ações referentes ao consumo que produzem impactos na triangulação formada por meio ambiente, sociedade e economia. É válido salientar que, em um panorama ideal de consumo consciente, o consumidor desenvolve ambos os aspectos - atitude e comportamento: atitude, sem a prática do consumo, não vai a lugar nenhum, enquanto apenas a ação ou compor- 
tamento, sem uma atitude consolidada, possui prazo de validade ou cessa, caso haja algum tipo de interferência.

Sabe-se que, no contexto brasileiro, já se destacam trabalhos acadêmicos em relação ao comportamento de compra de produtos verdes, como o de Marques (1998), que buscou entender quais as atitudes entre estudantes de Administração acerca do meio ambiente e como estas influenciam nos seus comportamentos de consumo; o de Hill (1999) que, mediante estudo de caso, mapeou motivações, dificuldades e benefícios de investimentos em produtos e serviços que contribuíssem para a preservação ambiental e como estes esforços são percebidos pelo consumidor; o de Rocha e Marques (2004), que realizou análise entre atitudes em prol do meio ambiente e o modo como estas se refletem no comportamento das pessoas na qualidade de consumidores, de modo a descobrir que, na maior parte das vezes, atitudes favoráveis a decisões de caráter ambiental não desencadeiam um respectivo comportamento de compra, além do fato de que mulheres, de maior nível de renda e instrução, são mais sensíveis aos apelos ambientais; o de Pereira e Ayrosa (2004), que investigou a influência do argumento ecológico e o seu impacto nas atitudes referentes à marca, embalagem e intenção de compra, concluindo que o apelo ecológico influencia positivamente apenas as atitudes em relação à marca, sendo indiferente à embalagem e intenção de compra entre estudantes de graduação e pós-graduação do Rio de Janeiro.

Tem-se, também, o trabalho de Rocha (2011), que apresentou o resultado de pesquisa sobre atitudes e comportamentos de compra de consumidores de diferentes tipos de bens com características ecológicas, havendo, ao fim, revelado o fato de que o valor atribuído aos produtos varia em função da sua tipologia; o de Matos e Buhamra (2010), que avaliam os componentes (afetivo, cognitivo e conativo) da atitude do consumidor em relação às características ecológicas nas embalagens e concluem que, malgrado as avaliações positivas acerca das atitudes em relação aos invólucros de mercadorias, isto não se reflete em um comportamento de compra compatível. Além destes, trabalhos como o de Gonçalves-Dias e Moura (2007) buscaram discutir a possibilidade de outro tipo de consumo mais sustentável, diferenciando as opções entre consumo verde e sustentável.

Um aspecto interessante demonstrado por Pickett et al. (1993) é que profissionais da área de Marketing e aqueles relacionados a políticas públicas devem atentar para a transferência de resultados entre um comportamento verde e outro. Desta forma, os autores ilustram, como exemplo, ao dizer que o consumidor que costuma reciclar seus produtos não é, necessariamente, o consumidor que trabalha mais para adquirir produtos ecologicamente corretos.

\subsection{Fatores situacionais: disponibilidade, preço e qualidade percebidos}

A despeito de altos níveis de atitude e intenção em prol do bem-estar ambiental terem sido registrados, tais fatos não acarretam a mesma intensidade no comportamento pró-ambiental (DUNLAP, SCARCE, 1991; TARRANT, CORDELLE, 1997). A discrepância ou gap entre atitudes e comportamentos ambientais advém do fato de as atitudes não conduzirem, necessariamente, a ações ambientalmente concordantes (GOSKEN; ADAMAN; ZENGINOBUZ, 2002; LAROCHE et al., 1996).

Wild (1995), ao perguntar aos seus entrevistados os principais entraves à compra de produtos verdes, descobriu que eram: a) decorrentes de problemas informacionais percebidos em entender o diferencial verde do produto; $b$ ) em razão do conflito percebido entre a vantagem em ter-se um produto verde e demais características do produto, como efetividade, conveniência de uso e preço; e c) em virtude de restrições situacionais, como a pressão de tempo e a disponibilidade de produtos. Dois anos depois, em pesquisa realizada por Wagner (1997), ratificou-se que, dentre as dificuldades encontradas para a realização da compra de produtos verdes, tem-se 
a importância percebida de algumas de suas características, tais como o preço, o desempenho e a conveniência, que tendem a afetar a tomada de decisão de compra verde negativamente.

D'Souza, Taghian e Khosla (2007) classificaram, ainda, o preço e a qualidade percebidos dos produtos em fatores situacionais (situational factors) que influenciam a tomada de decisão do consumidor, ou seja, atributos dos produtos verdes que são considerados no comportamento de compra.

Bonini e Oppenheim (2008) apresentam cinco grandes barreiras a serem sanadas entre as intenções e comportamentos dos consumidores quanto a produtos verdes. São elas: falta de crença nas reivindicações verdes, falta de consciência acerca dos produtos verdes, percepção negativa em relação à qualidade desses produtos, altos preços e baixa disponibilidade. Para sanar isso, deve-se, respectivamente, buscar uma relação honesta entre organização e consumidor, educar os consumidores, produzir produtos melhores, disponibilizar maior oferta de produtos e, ainda, trazer os produtos e serviços verdes para o alcance da comunidade em geral.

Quanto à questão do preço percebido, Jackson (2005) apontou o equilíbrio entre custos e benefícios como fator a ser considerado para o entendimento da discrepância entre atitude e ação. Comportamentos de consumo ecologicamente corretos demandam mais esforços do consumidor, tais quais: a aceitação de preços mais elevados e o desempenho ou qualidade inferior do produto. Essa declaração é convergente com o pensamento de Herzog (2009) ao informar que, malgrado os apelos ambientais cada vez mais frequentes, as pessoas ainda tendem a consumir seus produtos preferencialmente em razão do seu preço e qualidade. À medida que os consumidores consideram comum gastar suas economias para adquirir determinado produto, eles estão mais propensos a utilizar novos produtos verdes - inclusive aqueles que custam mais. Eles também se sentem mais confiantes para comprar um produto ou serviço quando entendem como isso pode ajudar o meio ambiente a médio e longo prazo, com base em suas atividades de consumo (BONINI; OPPENHEIM, 2008).

Quanto à qualidade percebida, nota-se que os consumidores continuam valorizando mais o desempenho, confiança e durabilidade do que um produto que baseie seu diferencial competitivo, simplesmente, no apelo emocional (CHURCHILL; PETER, 2003). Para transcender a qualidade como barreira, deve-se buscar uma imagem de produto que supere a tradicional.

A disponibilidade de produtos verdes também está entre os principais fatores que podem ocasionar o gap entre intenção e comportamento. De acordo com Dinato e Madruga (1999), os consumidores costumam perceber que não existe uma oferta adequada de produtos ecologicamente responsáveis no mercado e que faltam opções para que a pessoa escolha o que levar para casa. Ao decidir comprar produtos ecologicamente corretos, os consumidores ainda encontram um obstáculo: eles não conseguem encontrá-los. As empresas não podem vender seus produtos se os seus consumidores não os encontram nas prateleiras. Trata-se de uma afirmação óbvia, mas muitos dos produtos verdes do mercado não se encontram amplamente disponíveis e/ou distribuídos (BONINI; OPPENHEIM, 2008). A discussão acima conduz à hipótese:

H3 - A relação entre GPI e GPB é influenciada pela disponibilidade, preço e qualidade percebidos (SF).

Preço, qualidade e conveniência - entre outros diversos fatores - podem ser responsáveis pelo fato de a preocupação e a intenção de comportamento ambiental não acarretarem o comportamento de compra. 


\section{METODOLOGIA}

O estudo, em decorrência do seu paradigma positivista, é de natureza essencialmente quantitativa (COLLIS; HUSSEY, 2005). Tal abordagem foi escolhida, conforme sua nomenclatura, por buscar a quantificação e análise estatística dos dados (RICHARDSON, 2008; DIEHL; TATIM, 2004).

Quanto aos seus objetivos, trata-se de uma pesquisa descritiva e configura-se, ademais, como um estudo de corte transversal visto que as variáveis foram analisadas uma única vez, em momentos pontuais. No caso da presente pesquisa, foram aplicados dois questionários com um intervalo de um mês: o primeiro foi com elementos da escala GPA (Green Purchase Attitude) e GPI (Green Purchase Intention), apurando as atitudes e intenções dos consumidores e, posteriormente, o segundo com elementos da escala GPB (Green Purchase Behavior) e das afirmações relativas a disponibilidade, preço e qualidade percebidos (Situational Factors). Optou-se por esse intervalo de aplicação para observar se a atitude e intenção iriam, de fato, desencadear em um comportamento de compra, além de avaliar a influência dos fatores situacionais no caso estudado.

Quanto aos meios, a pesquisa é categorizada como bibliográfica (dados secundários) e de campo (dados primários). Bibliográfica, por ter sido baseada em material já desenvolvido, com sua fundamentação teórico-metodológica pautada em livros, jornais, periódicos e anais de eventos para a pesquisa dos assuntos relacionados à causa ambiental, à cultura, ao Marketing Verde, ao comportamento do consumidor verde, às suas atitudes, intenções e comportamentos de compra. Pesquisa de campo, pois, como ensina Godoy (1995), o fenômeno, para ser compreendido em sua totalidade, deve ser analisado em seu contexto e em âmbito integrado, exigindo que o pesquisador vá a campo para analisar o fenômeno estudado mediante a perspectiva das pessoas envolvidas. Houve, portanto, a coleta de dados primários de estudantes brasileiros de gestão.

Quanto ao método de levantamento de dados, foi aplicado um questionário padronizado, método comumente utilizado em pesquisas descritivas e que costuma garantir "a comparabilidade dos dados, aumentar a velocidade e a precisão do registro e facilitar o processamento de dados" (MALHOTRA, 2011, p. 290). O questionário de auto-aplicação foi formado por afirmações estruturadas, com um conjunto preestabelecido de respostas constituídas em escalas não comparativas e "itemizadas" por meio de escala de Likert de 7 pontos. Hair Jr. et al. (2005) esclarecem que é um formato baseado, geralmente, em descritores favoráveis e desfavoráveis e que é considerada uma escala intervalar pela maioria dos pesquisadores. Ademais, a aplicação foi realizada presencialmente, em sala de aula, nas duas ocasiões, devido ao índice de resposta ser maior do que se aplicado virtualmente, além de facilitar o acesso aos respondentes para o preenchimento da segunda fase de respostas relativas ao comportamento de compra e aos fatores situacionais.

A pesquisa foi aplicada com 220 universitários da área de gestão da Universidade Federal do Ceará (sendo, destes, 208 válidos) acerca de produtos verdes em maio e junho de 2012. Foi questionado, mais especificamente, acerca de produtos de conveniência, por estes serem facilmente encontrados a baixos custos, possuírem alta frequência de compra e estarem subdivididos em situações de necessidade, impulso e emergência (KOTLER; KARTSJAY; SETIAWAN, 2010). Tal escolha torna mais provável o fato de o respondente ir às compras durante o mês intervalar da pesquisa de campo, além de ser o tipo de produto eleito por Chan (2001), um dos trabalhos nos quais o estudo é sustentado.

Para a análise dos dados coletados, optou-se pela utilização da Modelagem em Equações Estruturais (MEE) ou Structural Equation Modeling (SEM). Essa técnica corresponde a um conjunto de procedimentos estatísticos que visa analisar as relações entre uma ou mais variáveis independentes e uma ou mais variáveis dependentes. Isto é, de acordo com Pilati e Laros (2007, 
p. 206-207), trata-se de "uma técnica de equações estruturais ou equações múltiplas, mas diferencia-se da análise de trajetórias porque trabalha com variáveis latentes, ou seja, medidas não diretamente observadas, mas sim derivadas de indicadores observáveis", pressupondo a normalidade das variáveis. Além disso, é válido ressaltar que para a mensuração do construto GPA (atitude), GPI (intenção) e GPB (comportamento), foram atribuídas seis variáveis observáveis. No caso do construto SF (fatore situacionais), foram relacionadas três variáveis.

Os procedimentos estatísticos referentes à análise multivariada dos dados foram realizados mediante o uso dos softwares estatísticos Statistical Package for the Social Sciences (SPSS) 20.0 e Analysis of Moment Structures (AMOS) 20.0.

\section{APRESENTAÇÃO E ANÁLISE DOS RESULTADOS}

O perfil dos respondentes, revelado a partir dos dados sociodemográficos referentes à amostras de alunos, indicou que a maioria é composta por homens (56,08\%), entre 21 e 25 anos $(78 \%)$, solteiros $(85,2 \%)$, que trabalha ou estagia meio período $(66,7 \%)$, com renda individual entre 1 e 2 salários mínimos (41\%) e renda familiar entre 6 e 10 salários mínimos (34\%).

Em um primeiro momento, foi analisado, por meio da estatística univariada, o Alpha de Cronbach de cada construto para avaliar a sua consistência interna. Hair et al. (2005) recomendam o Alpha de Cronbach por este ser o índice mais prático e comum para a análise de consistência interna e recomendam ainda um valor mínimo e aceitável de 0,70. GPA, GPI, GPB e SF apresentaram valores maiores (respectivamente 0,$74 ; 0,89 ; 0,92 ;$ e 0,72 ).

Posteriormente, com a delimitação dos construtos baseada na teoria empreendida, seguiu-se para o desenvolvimento dos modelos de mensuração e estrutural. Por meio destes modelos, foram estabelecidas as relações causais entre os construtos (variáveis latentes) e os seus respectivos indicadores (variáveis de medida). O passo seguinte referiu-se à validação individual dos construtos e à validação do modelo integrado.

É válido ressaltar que, ao utilizar a Modelagem em Equações Estruturais, deve-se obter um mínimo de 100 de respondentes ou respostas válidas, apesar de que o número total de segurança deveria ser maior do que 200 (MARSH et al., 1998; HAIR et al., 2005). Dessa forma, observa-se que o tamanho total da amostra, para a validação do modelo integrado, está de acordo com o número solicitado pelos autores e que isso não seria um limitante para a atual pesquisa, visto que se têm, no total, 208 casos. Além disso, foi utilizada a técnica de Máxima Verossimilhança (MLE) para estimação dos coeficientes, na qual se requer que haja em torno de 100 a 200 casos e que não ultrapasse de 400 a 500, visto que, a partir dessa quantidade, as medidas de qualidade de ajuste podem ser prejudicadas (HAIR JR et al., 2005; SCHUMACKER; LOMAX, 2004). Por meio dessa técnica, foram substituídos os missing values ou valores faltantes pela média.

$\mathrm{O}$ teste de validação dos construtos individuais presentes no modelo foi realizado com um total de 208 respondentes. Com a utilização da Análise Fatorial Confirmatória (AFC), prosseguiu-se com a análise e interpretação dos dados para o ajustamento, com o intuito de melhorar o modelo geral.

Dessa forma, calculou-se a carga fatorial padronizada, $t$-value e erro da relação de cada construto com suas variáveis de medida. Os índices de confiabilidade composta e de variância extraída foram utilizados para aferir a confiabilidade. Ademais, têm-se os valores de ajustamento referente ao construto a partir dos índices expostos na metodologia, tais quais: RMSEA, GFI, TLI, $\mathrm{AGFI}, \mathrm{\chi} 2 / \mathrm{gl}, \mathrm{NFI}, \mathrm{CFI}$ e AIC.

Com a verificação da confiabilidade e validade dos construtos, pôde-se ter uma condição mais refinada destes e, assim, seguir para a validação do modelo integrado. 
Para melhor identificar os dados a serem analisados, apresentam-se, no Quadro 1, os construtos utilizados na Modelagem em Equações Estruturais, assim como os respectivos códigos e indicadores.

Quadro 1 - Nomes, códigos e indicadores dos construtos utilizados na AFC

\begin{tabular}{|c|c|c|}
\hline $\begin{array}{l}\text { Construto } \\
\text { (variável } \\
\text { latente) }\end{array}$ & Código & Indicador (variável de medida) \\
\hline \multirow[t]{6}{*}{ GPA } & AT1 & Eu (1= não gosto; 7= gosto) da ideia de comprar produtos verdes \\
\hline & AT2 & Comprar produtos verdes é uma (1= má; $7=$ boa) ideia \\
\hline & AT3 & $\begin{array}{l}\text { Eu tenho uma atitude ( } 1=\text { desfavorável; } 7=\text { favorável) em relação à compra da ver- } \\
\text { são verde de um produto }\end{array}$ \\
\hline & AT4 & $\begin{array}{l}\text { Quando eu sei dos possíveis danos que um produto pode causar ao meio ambiente, eu } \\
\text { tento limitar o seu uso ( } 1=\text { discordo totalmente; } 7=\text { concordo totalmente). }\end{array}$ \\
\hline & AT5 & $\begin{array}{l}\text { Eu me sinto triste/chateado quando penso nos danos que o desperdício de produtos } \\
\text { tem causado às plantas e animais ( } 1=\text { discordo totalmente; } 7=\text { concordo totalmente). }\end{array}$ \\
\hline & AT6 & $\begin{array}{l}\text { Eu tento convencer meus amigos e família a evitarem produtos prejudiciais ao } \\
\text { meio ambiente ( } 1=\text { discordo totalmente; } 7=\text { concordo totalmente). }\end{array}$ \\
\hline \multirow[t]{6}{*}{ GPI } & INT1 & $\begin{array}{l}\text { Durante o próximo mês, eu considerarei comprar produtos por eles serem menos } \\
\text { poluentes }\end{array}$ \\
\hline & INT2 & Durante o próximo mês, eu considerarei trocar de marca devido a razões ecológicas \\
\hline & INT3 & Durante o próximo mês, eu planejo trocar para a versão verde de um produto \\
\hline & INT4 & $\begin{array}{l}\text { Durante o próximo mês, eu considerarei comprar produtos com embalagens reci- } \\
\text { cláveis e/ou biodegradáveis }\end{array}$ \\
\hline & INT5 & Durante o próximo mês, eu planejo comprar produtos recicláveis, reutilizáveis ou em refil \\
\hline & INT6 & $\begin{array}{l}\text { Durante o próximo mês, eu considerarei comprar produtos com selo verde (Certi- } \\
\text { ficação ISO, por exemplo) }\end{array}$ \\
\hline \multirow[t]{6}{*}{ GPB } & $\mathrm{BH} 1$ & Com que frequência comprou produtos verdes no último mês? \\
\hline & $\mathrm{BH} 2$ & Quanto gastou em produtos verdes no último mês? \\
\hline & $\mathrm{BH} 3$ & Quantos produtos verdes comprou no ultimo mês? \\
\hline & $\mathrm{BH} 4$ & Frequência de compra de produtos com embalagens recicláveis ou biodegradáveis \\
\hline & $\mathrm{BH} 5$ & Frequência de compra de produtos recicláveis, reutilizáveis ou em refil \\
\hline & $\mathrm{BH} 6$ & Frequência de compra de produtos com selos verdes (Certificado ISO, por exemplo) \\
\hline \multirow[t]{3}{*}{ SF } & A & Disponibilidade \\
\hline & PP & Preço percebido \\
\hline & $\mathrm{PQ}$ & Qualidade percebida \\
\hline
\end{tabular}

Fonte: Dados da pesquisa.

Ademais, é necessário alertar que, para os construtos GPA, GPI e GPB, foram incluídas covariâncias entre os erros do mesmo construto, visto que foram sugeridos pelo AMOS após a AFC e que tal modificação resultou nos melhores índices. Sabe-se que, qualquer alteração nos construtos e no modelo deve ser amparada pela teoria subjacente a eles. No caso, Hair et al. (2005) informam que é possível que variáveis de medida (VM) de um mesmo construto compartilhem de covariâncias entre os erros das variáveis. Ademais, os três construtos apresentavam consistente relação teórica entre si, o que também justifica as covariâncias indicadas pelo AMOS.

Após a validação e estimação dos construtos no plano individual, seguiu-se para a validação do modelo integrado, com amparo na técnica de Modelagem em Equações Estruturais, proposto por este estudo. Nesta subseção, apresentam-se os testes de validade discriminante e multicolinearidade para validar o modelo de mensuração e, para o modelo estrutural, utilizam-se as medidas de ajustamento entre as variáveis e o teste de hipóteses. 
O modelo integrado é composto por um total de 25 variáveis, das quais quatro variáveis são latentes (VL) e 21 são variáveis de medida (VM) ou indicadores. Todas as variáveis presentes no modelo podem ser observadas na figura 1, que mostra, simultaneamente, o modelo de mensuração (relações entre os construtos e seus indicadores) e o modelo estrutural (relações entre os construtos) com base na teoria e com a incorporação das covariâncias entre os erros de algumas variáveis de medida (VM):

Figura 1 - Modelo estrutural e de mensuração proposto

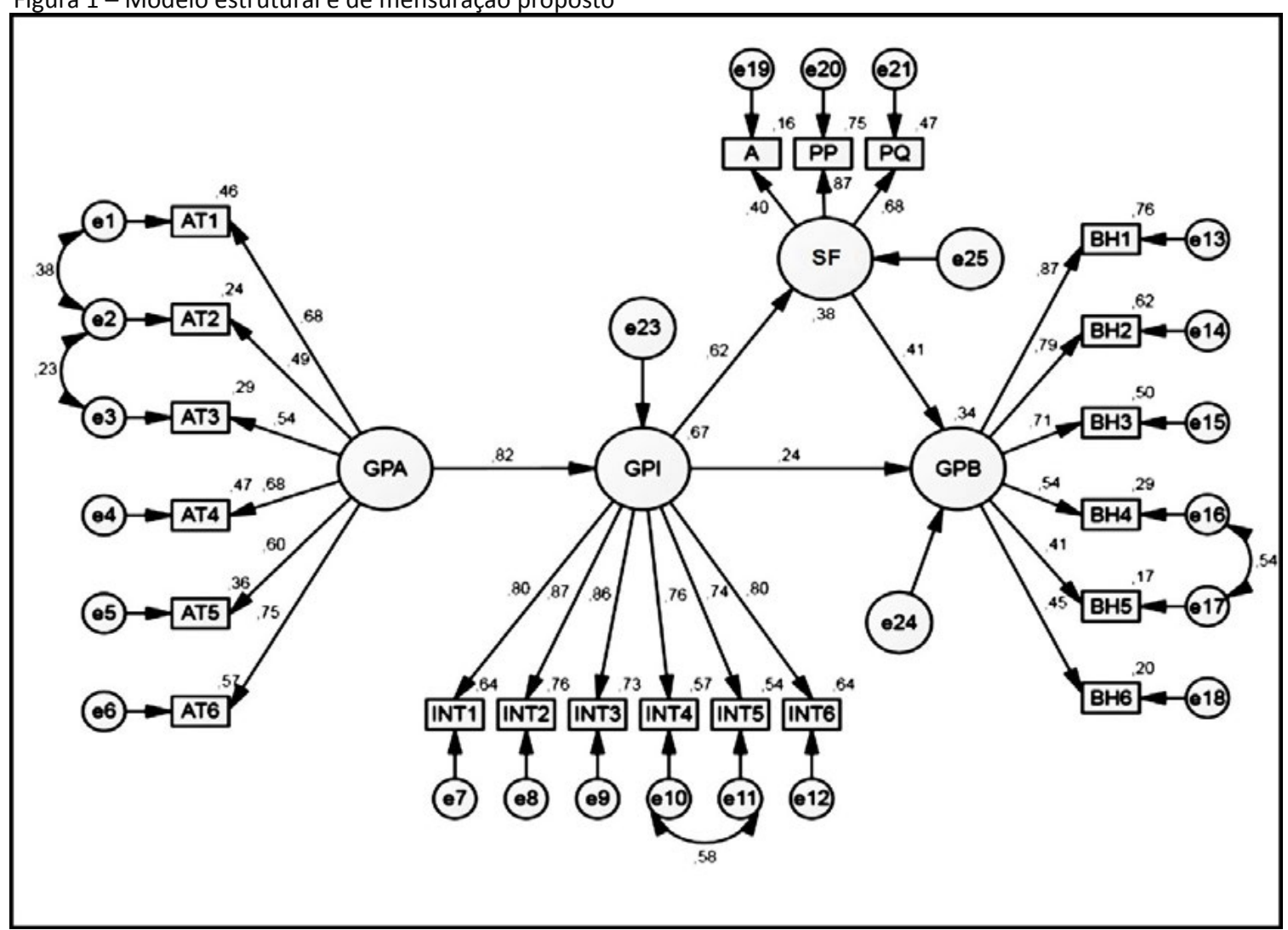

Fonte: Dados da pesquisa com base no output gráfico gerado pelo software AMOS 20.0.

No caso da validade discriminante deste estudo, todas as variâncias extraídas mostraram valores maiores do que as variâncias compartilhadas, concluindo que cada construto possui uma escala que "é suficientemente diferente de outros conceitos semelhantes para ser distinta" (HAIR et al., 2005, p.112).

A multicolinearidade, que deve ser rejeitada, isto é, possuir todos os valores de intercorrelações abaixo de 0,85, de acordo com Kline (1998), ou até 0,90 (TABACHNIK; FIDEL, 2001), está representada na tabela 1 :

Tabela 1 - Inter-correlações entre os construtos do modelo

\begin{tabular}{c|c|c|c|c}
\hline & GPA & GPI & SF & GPB \\
\hline GPA & 1,000 & & & \\
\hline GPI & 0,830 & 1,000 & & \\
\hline SF & 0,580 & 0,604 & 1,000 & \\
\hline GPB & 0,408 & 0,532 & 0,780 & 1,000 \\
\hline
\end{tabular}

Fonte: Dados da pesquisa. 
Nota-se que a intercorrelação mais alta está é observada na relação entre GPA (Atitude) e GPI (Intenção), com o valor 0,830 , o que indica, apesar de próximo ao limite, que não existe multicolinearidade entre os construtos do modelo.

Após a validação do modelo de mensuração, destaca-se a necessidade de verificar o modelo estrutural. Dessa forma, realiza-se o teste de medidas de ajustamento do modelo integrado, seguido do teste de hipóteses.

Para análise e validação do modelo estrutural, estimaram-se os valores referentes aos índices RMSEA, GFI, TLI, AGFI, $\chi 2 / g l, N F I, T L I$ e AIC, conforme exibidos na tabela 2:

Tabela 2 - Medidas de ajustamento do modelo integrado

\begin{tabular}{c|c|c|c|c|c|c|c|c|}
\hline $\begin{array}{c}\text { Modelo Inte- } \\
\text { grado }\end{array}$ & $\begin{array}{c}\text { RMSEA } \\
<0,08\end{array}$ & $\begin{array}{c}\text { GFI } \\
>0,80\end{array}$ & $\begin{array}{c}\text { TLI } \\
>0,90\end{array}$ & $\begin{array}{c}\text { AGFI } \\
>0,80\end{array}$ & $\begin{array}{c}\chi 2 / g I \\
<5\end{array}$ & $\begin{array}{c}\text { NFI } \\
>0,90\end{array}$ & $\begin{array}{c}\text { TLI } \\
>0,90\end{array}$ & $\begin{array}{c}\text { AIC } \\
>0,80\end{array}$ \\
\cline { 2 - 10 } & 0,042 & 0,87 & 0,93 & 0,82 & 1,815 & 0,84 & 0,92 & 1530,342 \\
\hline
\end{tabular}

Fonte: Dados da pesquisa.

Nota-se que quase todos os índices estão com valores satisfatórios para o modelo e que este foi considerado ajustado com apoio nas validações realizadas em seus modelos de mensuração (análise discriminante e multicolinearidade) e estrutural (teste de medidas de ajustamento). Seguiu, portanto, para o teste de hipóteses.

Os modelos representados por MEE, de modo geral, significam uma série de relações hipotéticas de causa e efeito que, quando determinadas e interpretadas, permitem a confirmação ou não de hipóteses, com base em dados estatísticos.

Kline (1998) esclarece que as hipóteses são confirmadas caso o parâmetro estimado para cada caminho do modelo estrutural seja significativo, isto é, com o t-value $>1,96$ para $p<0,05$ e $>2,33$ para $p<0,01$. A tabela 3 define, portanto, os parâmetros estimados do modelo estrutural e o teste de hipóteses.

Tabela 3 - Parâmetros Estimados do Modelo Integrado e Teste das Hipóteses

\begin{tabular}{c|c|c|c|c}
\hline Relação & $\begin{array}{c}\text { Carga fatorial padro- } \\
\text { nizada }\end{array}$ & $\begin{array}{c}\text { Co-variância } \\
\text { (t-value) }\end{array}$ & Hipótese & Resultado \\
\hline $\mathrm{GPA} \rightarrow \mathrm{GP}$ & $0,821(4,842)^{* * *}$ & & $\mathrm{H} 1$ & Não rejeitada \\
\hline $\mathrm{GPI} \rightarrow \mathrm{GPB}$ & $0,243(3,258)^{* *}$ & & $\mathrm{H} 2$ & Não rejeitada \\
\hline $\mathrm{GPI} \rightarrow \mathrm{SF}$ & $0,627(6,101)^{* * *}$ & & $\mathrm{H} 3$ & Não rejeitada \\
\hline $\mathrm{SF} \rightarrow \mathrm{GPB}$ & $0,412(4,390)^{* * *}$ & & - & - \\
\hline $\mathrm{e} 1-\mathrm{e} 2$ & & $0,384(3,392)^{* * *}$ & - & - \\
\hline $\mathrm{e} 2-\mathrm{e} 3$ & & $0,234(2,389)^{* * *}$ & - & - \\
\hline $\mathrm{e} 10-\mathrm{e} 11$ & & $0,582(5,678)^{* * *}$ & - & - \\
\hline
\end{tabular}

Fonte: Dados da pesquisa.

$* * * p<0,001 * * p<0,05$

A hipótese 1, que pressupôs que a relação entre atitude de compra de produtos verdes (GPA) está positivamente relacionada à intenção de compra desses produtos (GPI), foi confirmada. Tal resultado é amparado pela Teoria da Ação Racional (TAR), ao afirmar que, quanto mais favorável for a atitude em relação ao objeto, maior será, também, a sua intenção comportamental (AJZEN; FISHBEIN, 1980; FISHBEIN; AJZEN, 1975). De acordo com Laroche, Bergeron e Barbaro-Forleo (2001), as atitudes são as preditoras mais importantes quanto à disposição de pagar mais por produtos verdes.

A satisfatoriedade dos índices, além da carga fatorial significativa entre os construtos e seus indicadores, consolida a forte relação $(0,821)$ encontrada no caminho GPA $\rightarrow$ GPI, o que é novamente ratificado por Laroche, Bergeron e Barbaro-Forleo (2001) ao afirmarem que, quanto maior o conhecimento acerca de questões ambientais, maior será a atitude/propensão à compra de produtos verdes. 
A hipótese 2, que afirma haver uma relação positiva entre a intenção de compra de produtos verdes (GPI) e a efetivação desse comportamento de compra (GPB), isto é, GPI $\rightarrow$ GPB positiva, também foi confirmada. Apesar do índice inferior $(0,243)$, se comparado aos demais caminhos, pode-se afirmar que se trata de uma relação positiva e significativa. Este resultado está de acordo com Ajzen e Madden (1986), quando revelam que o imediato antecedente de qualquer comportamento é a intenção de realizá-lo: quanto maior a intenção da pessoa, mais se espera que ela tente, e, portanto, maior será a probabilidade do comportamento ser realizado. Apesar das possíveis diferenças encontradas, pesquisas empíricas apontam que as variáveis: intenção e comportamento demonstram altos níveis de correlação. As intenções dos consumidores contribuem, portanto, sobremaneira, para a elaboração de estratégias de mercado, pois elas tendem a atuar como uma previsão do comportamento (BLACKWELL; MINIARD; ENGEL, 2005).

A hipótese 3, referente à afirmação de que "o gap existente entre GPI e GPB é influenciado pela disponibilidade, preço e qualidade percebidos", isto é, que a variável SF - como moderadora da relação entre $\mathrm{GPI} \rightarrow \mathrm{GPB}$ - influencia esse caminho de forma significativa, também foi confirmada. Enquanto GPI $\rightarrow$ SF possui uma carga explicativa de $0,697, \mathrm{SF} \rightarrow \mathrm{GPB}$ demonstra possuir carga equivalente de 0,622 , o que assevera a influência da disponibilidade, preço e qualidade percebidos nessa relação entre intenção e comportamento. A existência desse gap pode ainda ser considerada como justificativa para o baixo índice (ainda que positivo) da relação GPI $\rightarrow$ GPB $(0,243)$ visto que, quando a variável moderadora SF é retirada do modelo, a relação entre intenção e comportamento aumenta para 0,670, de forma positiva e significativa. Tal procedimento é amparado por Shrout e Bolger (2002), quando explicam que o efeito de uma variável (no caso, o GPI) em outra variável (como GPB) enfraquece no momento no qual é adicionada a variável moderadora, de modo a não controlar-se GPI e GPB.

Ajzen e Madden (1986) demonstraram que vários fatores podem interferir no comportamento pretendido, sejam eles internos (como habilidades, conhecimento e planejamento) ou externos (tempo, oportunidade e dependência de terceiros). Na verdade, os autores garantem que não se pode ter certeza de que as pessoas possuem os recursos necessários ou a oportunidade apropriada para a concretização do comportamento, até que ele seja realmente efetivado. Motta e Rossi (2003) são enfáticos, acentuando que, dentre os motivos mais evidentes para que a atitude não se transforme em comportamento de compra, tem-se a falta de informação e oferta para tal. Ademais, a qualidade percebida do produto está intimamente relacionada à satisfação do consumidor, o que a torna um importante fator no momento da decisão de compra. Assinala-se, portanto, na literatura acerca dos estímulos e influenciadores no comportamento e compra, características tais quais qualidade, preço e disponibilidade como importantes fatores na relação entre a intenção e a efetivação da compra (HOWARD; SHETH, 1969; BLACKWELL; MINIARD; ENGELL, 2005).

$\mathrm{Na}$ fase acerca da validação do construto, tornaram-se notórias as intensas relações estabelecidas entre a variável latente e suas respectivas variáveis de medida, sendo a SF $\rightarrow$ PP (preço percebido) a mais forte entre elas. O bom ajustamento do construto, assim como sua unidimensionalidade e confiabilidade composta, também auxiliara na consolidação da variável como moderadora da intenção e compra de produtos verdes da amostra geral.

\section{CONSIDERAÇÕES FINAIS}

A compreensão sobre atitude, intenção e comportamento de compra de produtos verdes tende a gerar debates e discussões acerca das vantagens (e desvantagens) na adoção de produtos verdes, assim como dos papéis das organizações na disponibilização destes. Ao entender 
a importância de um planeta mais justo e equilibrado, torna-se essencial, por parte do cidadão e consumidor, manter-se informado mediante a busca de informações alheias a vieses para que possa eleger o que e por que consumir. Dessa forma, espera-se que o leitor encontre, aqui, um arcabouço teórico e empírico que possa auxiliá-lo nessa formação.

Ademais, o estudo pode contribuir para gestores que irão elaborar sistemas de gestão ambiental corporativo em grandes e pequenas empresas, assim como para profissionais da área de Marketing que buscam desenvolver estratégias com base no comportamento de seus consumidores (BRANZEl et al., 2001). De forma a integrar implicações acadêmicas e empresariais, propõe-se uma mudança gradual na educação, com suporte na técnica de currículos gerenciais, 0 que poderá corresponder às novas necessidades do mercado de trabalho e, consequentemente, as exigências para especialistas estarão satisfeitas com os administradores necessários enquanto principal fator de mudança para a formulação de novas políticas e gestão.

Espera-se, por fim, que ao final deste estudo tenha-se estimulado o surgimento de pesquisas inovadoras que permitam analisar as complexidades presentes no comportamento de consumo verde, assim como ampliar e consolidar a discussão da sustentabilidade na área do Marketing, seja em meio acadêmico ou empresarial. Ao estudar construtos como atitude, intenção, comportamento de compra, cultura, disponibilidade, preço e qualidade percebidos, esperou-se contribuir com o atual conhecimento acerca dessas variáveis, de modo a fornecer base bibliográfica e conceitual para demais trabalhos sobre o tema.

\section{REFERENCIAS}

AAKER, D. A.; MYERS; J. G. Advertising Management. New York: Prentice- Hall, 1987.

AFONSO, A. C. B. O Consumidor Verde: Perfil e Comportamento de Compra. 2010. 117 p. Dissertação (Mestrado em Marketing) Instituto Superior de Economia e Gestão, Universidade Técnica de Lisboa, 2010.

AJZEN, I. Consumer attitudes and behavior. In: HAUGTVEDT, C. P.; HERR, P. M.; CARDES, F. R. (Eds.), Handbook of Consumer Psychology, p. 525- 548, 2008.

AJZEN, I.; FISHBEIN, M. Understanding atitudes and predicting social behavior. Englewood Cliffs, NJ: Prentice-Hall, 1980.

AJZEN, I.; MADDEN, J. T. Prediction of goal directed behaviour; Attitudes, intentions and perceived control. Journal of Experimental Social Psychology. 22, 253-274, 1986.

ALI, A.; KHAN, A. A.; AHMED, I. Determinants of Pakistani Consumers' Green Purchase Behavior: Some Insights from a Developing
Country. International Journal of Business and Social Science, 2(3), January 2011.

ALMEIDA JÚNIOR, J. M. G. Desenvolvimento ecologicamente auto-sustentável: conceitos, princípios e implicações. Humanidades, 10, p. 284-299, 1994.

ALWITT; L. F.; PITTS; R.E. Predicting purchase intentions for an environmentally-sensitive product. Journal of Consumer Psychology, n. 5, v. 1, p. 49-64, 1996.

BERKMAN, H. W.; GILSON; C. C. Consumer behavior: Concepts and strategies. California: Dickenson Publishing Company Inc, 1979.

BLACKWELL, R. D.; MINIARD, P. W.; ENGEL, J. F. Comportamento do Consumidor. 9a ed. São Paulo: Pioneira Thomson Learning, 2005.

BONINI, S.; OPPENHEIM, J. Cultivating the Green Consumer. Stanford Social Innovation Review, Fall 2008.

BRANZEI, O.; VERNISTKY, I.; TAKAHASHI, T.; ZHANG, W. Corporate Environmentalism across Cultures. A Comparative Field Study of Chinese and Japanese Executives. Cross Cultural 
Management, v. 1, n. 3, p. 287-312, 2001.

CHAN, R. Y. K. Determinants of Chinese Consumers' Green Purchase Behavior. Psychology \& Marketing, v 18, n. 4, p 389-413, 2001.

CHURCHILL, G. A. Jr.; PETER, P. J. Marketing criando valor para os clientes. 2. ed. São Paulo: Saraiva, 2003.

COLLIS, J.; HUSSEY, R. Pesquisa em Administração: um guia prático para alunos de graduação e pós-graduação. 2a ed. Porto Alegre: Bookman, 2005.

CONFEDERATION OF BRITISH INDUSTRY. Climate Change: Everyone's Business. A Summary Report from the CBI Climate Change Task Force. London: CBI, 2007.

CORDANO, M.; WELCOMER, S.; SCHERER; R. F.; PRADENAS, L.; PARADA, V. A CrossCultural Assessment of Three Theories of Pro-Environmental Behavior: A comparison Between Business Students of Chile and the United States. Environment and Behavior, 43, 634-657, 2010.

CORRAL-VERGUDO, V.;ARMENDARIZ, L. I. The "New Environmental Paradigm" in Mexican community. Journal of Environmental Education, 31, p. 25-31, 2000.

CRONIN, J. J.; BRADY, M. K.; HULT, G.T.M. Assessing the effects of quality, value, and customer satisfaction on consumer behavioral intentions in service environments. Journal of Retailing, v. 76, n. 2, p. 193-218, 2000.

DERKSEN, L.; GARTRELL, J. The Social Context of Recycling. American Sociological Review, $\mathrm{n}$ 58, p. 434-442, 1993.

DIAMANTOPOULOS, A.; SCHLEGELMICH, B. B.; SINKOVICS, R. R.; BOHLEN, G. M. Can sociodemographics play a role in profiling green consumers? A review of the evidence and an empirical investigation. Journal of Business Research, 56(2), p. 465-80, 2003.
DIAS, R. Marketing ambiental: ética, responsabilidade social e competitividade nos negócios. São Paulo: Atlas, 2008.

DIEHL, A. A.; TATIM, D. C. Pesquisa em ciências sociais aplicadas. São Paulo: Prentice Hall, 2004.

DINATO, M; MADRUGA, K. Technological innovation and performance of the ecological products in the Brazilian market: a multisectorial analysis. In: Seventh International Conference on Management of Technology Annals of International Association for the Management of Technology, 1999.

DONAIRE, D. A internalização da gestão ambiental. Revista de Administração de

Empresas. V. 31, p. 44-51, 1996.

D'SOUZA, C.; TAGHIAN, M.; KHOSLA, R. Examination of environmental beliefs and its impact on the influence of price, quality and demographic characteristics with respect to green purchase intention. Journal of Targeting, Measurement and Analysis for Marketing, v. 15, p. 69-78, 2007.

DUNLAP, R. E; SCARCE, R. The Polls-Poll Trends: Environmental Problems and

Protection. Public Opinion Quarterly, v. 55, p. 651-672, 1991.

FISHBEIN M.; AJZEN, I. Belief, Attitude, Intention and Behavior: An introdution to theory and research. Reading, Mass: Adisson-Wesley, 1975.

FISHBEIN M.; AJZEN, I. Understanding attitudes and predicting social behaviour. Englewood Cliffs, New Jersey: Prentice-Hall, 1980.

FRANZEN, A.; MEYER, R. Environmental Attitudes in Cross-National Perspective: A Multilevel Analysis of the ISSP 1993 and 2000. European Sociological Review, v. 26, n. 2, p. 219-234, 2010.

GHAHREMANI, Y. European firms expanding environmental management. Business International, v.37, n.40, p.333-338, 1990. 
GODOY, A. S. Introdução à pesquisa qualitativa e suas possibilidades. Revista de Administração de Empresas, Rio de Janeiro, 35 (2), p. 57-63, mar./abr., 1995

GOLDEMBERG, J.; VILLANUEVA, L. D. Energia, meio ambiente \& desenvolvimento. São Paulo: Edusp, 2003.

GONÇALVES-DIAS, S. L. F.; MOURA, C. Consumo Sustentável: Muito Além do Consumo "Verde". In: XXXI Enanpad - Encontro dos programas de pós-graduação em Administração, 2007, Rio de Janeiro. Anais do XXXI Enanpad, 2007.

GOSKEN, F.; ADAMAN, F.; ZENGINOBUZ, E. On environmental concern, willingness to pay and post-materialist values: evidence from Istanbul. Environment and Behavior, v. 34, n. 5, p 533-616, 2002.

GUIMARÃES, R. P. O novo padrão de desenvolvimento para o Brasil: inter-relação do desenvolvimento industrial e agrícola com o meio ambiente. In: VELLOSO, J. P. R. et al. (Orgs.). A ecologia e o novo padrão de desenvolvimento no Brasil. São Paulo: Nobel, 1992.

HAIR JR., J. F.; ANDERSON, R. E.; TATHAM, R. L.; BLACK, W. C. Análise multivariada de dados. 5. ed. Porto Alegre: Bookman, 2005.

HERZOG, A. L. Fazer mais, falar menos. Revista EXAME. Ed. 951, 09 set 2009.

HILL, A. C. As motivações da utilização do Marketing Verde como estratégia empresarial. (Dissertação de Mestrado) Departamento de Administração, Pontifícia Universidade Católica do Rio de Janeiro, 1999.

HOBSBAWM, E. Age of Extremes. The short twentieth century 1914-1991. London: Penguin, 1994.

HOWARD, J.; SHETH, J. An Experiment on Consumer Dissonance. Journal of Marketing, $v$. 31, n. 1, p. 39-43, 1969.
JACKSON, T. Live Better by Consuming Less: is there a double dividend in sustainable consumption. Journal of Industrial Ecology, v. 9, n 1-2, p. 19-36, 2005.

KINNEAR, T. C; TAYLOR, J. R. The effect of ecological concern on brand perceptions. Journal of Marketing Research, v. 10, p. 191197, May 1973.

KLINE, R. B. Principles and practice of structural equation modeling. New York, Guilford: 1998.

KOTLER, P.; KARTSJAYA, H.; SETIAWAN, I. Marketing 3.0: From Products to Costumers to the Human Spirit. Hoboken, New Jersey: John Wiley \& Sons, 2010.

LAMBIN, J. Marketing estratégico. 40 ed. Madrid: McGraw- Hill, 2002.

LAROCHE, M.; BERGERON, J.; BARBAROFORLEO, G. Targeting consumers who are willing to pay more for environmentally friendly products. Journal of Consumer Marketing, v. 18, n. 6, p. 503-520, 2001.

LAROCHE, M.; TOFFOLI, R.; KIM, C.; MULLER, T. E. The Influence of Culture on Proenvironmental Knowledge, Attitudes, and Behavior: a canadian perspective. Advances in Consumer Research, v. 23, p. 196-202, 1996.

LEONIDOU, C. N.; LEONIDOU, L. C. Research into environmental marketing/management: a bibliographic analysis. European Journal of Marketing , 45, 68-103, 2011.

MAIMON, D. Eco-estratégia nas empresas brasileiras: realidade ou discurso? Revista de Administração de Empresas, v.34, n.4, p.119130, 1994.

MALHOTRA, N. K. Pesquisa de marketing: uma orientação aplicada. 3. ed. Porto Alegre: Bookman, 2011.

MALHOTRA, N. K.; MCCORT, J. D. A cross-cultural comparison of behavioral intention models: Theoretical consideration and an empirical 
investigation. International Marketing Review, v. 18, n.3, p. 235-269, 2001.

MARQUES, D. L. de S. Eco-Atitudes vs EcoComportamentos: Os Reflexos da Atitude Ecológica no Comportamento do Consumidor. Dissertação de Mestrado. Departamento de Administração. Pontifícia Universidade Católica do Rio de Janeiro, 1998.

MARSH, H. W.; HAU, K. T.; BALLA, J. R.; GRAYSON, $D$. Is more ever too much? The number of indicators per factor in confirmatory factor analysis. Multivariate Behavioral Research, v. 33, p. 181-220, 1998.

MATOS, B. G.; BUHAMRA, C. A atitude do consumidor em relação às características ecológicas das embalagens. In: ENCONTRO INTERNACIONAL SOBRE GESTÃO EMPRESARIAL E MEIO AMBIENTE, 12., São Paulo. Anais... São Paulo: ENGEMA, 2010.

MENON, A.; MENON, A. Enviropreneurial marketing strategy: the emergence of corporate environmentalism as marketing strategy. Journal of Marketing, v. 61, p. 51-67, January 1997.

MOSTAFA, M. M. Antecedents of Egyptian Consumers' Green Purchase Intentions'. Journal of International Consumer Marketing, v. 19, n. 2, p. 97-126, 2006.

MOTTA, S. L. S.; ROSSI, G. B. A Influência do Fator Ecológico na Decisão de Compra de Bens de Conveniência. Revista de Administração da USP, v. 38, n. 1, p. 46-57, 2003.

MOWEN, J. C.; MINOR, M. S. Comportamento do consumidor. São Paulo: Prentice Hall, 2003.

NASCIMENTO, L. F. M.; TREVISAN, M.; FIGUEIRÓ, P.S.; BOSSLE, M. B. Do Consumo ao Descarte de Produtos e Embalagens: Estamos alienados? Rev. Adm. UFSM, Santa Maria, v. 7, n. 1, p. 33-48, mar. 2014.

OTTMAN, J. Marketing Verde: desafios e oportunidades para a nova era do marketing. São Paulo, Makron Books, 1993.
PEREIRA, S. J. N.; AYROSA, E. A. T. Atitudes relativas a marcas e argumentos ecológicos: um estudo experimental. Revista Eletrônica de Gestão Organizacional, v. 2, n. 2, p. 134- 145, 2004.

PICKETT, G. M.; KANGUN, N; GROVE, S. J. Is There a General Conserving Consumer? A Public Policy Concern. Journal of Public Policy and Marketing, 12, 234-243, february 1993.

PILATI, R.; LAROS, J. A. Modelos de Equações Estruturais em Psicologia: Conceitos e Aplicações, Psicologia: Teoria e Pesquisa, v. 23, n. 2, p 205-216, 2007.

POLONSKY, M. J.; ROSEMBERGUER III, P. J. Reevaluating Green Marketing: a strategic approach. Business Horizons. September-October 1995.

REYES-RICON, M. Conhecimento e Afeto Ecológico: Antecedentes do Consumo Ecológico. 2010. 107 p. Dissertação (Mestrado em Administração Pública) - Escola Brasileira de Administração Pública e de Empresa, Fundação Getúlio Vargas, São Paulo, 2010.

RICHARDSON, R. J. et al. Pesquisa Social: métodos e técnicas. 3. ed. revista e ampliada. São Paulo: Atlas, 2008.

ROCHA; A. L. Intenções e Ações em Relação a Escolhas de Produtos Ecológicos: Estudo sobre o Comportamento do Consumidor Carioca. Revista Contemporânea de Economia e Gestão, 9(1), jan/jun 2011.

ROCHA, A. L. P; MARQUES, D. L. C. Marketing Verde Para Quem? ENANPAD, 28. In: Anais... ANPAD, 2004.

RODRIGUES, A. Psicologia Social. 5a.ed.: Vozes, Petrópolis, 1976.

RODRIGUES, A.; ASSMAR, E. M. L.; JABLONSKI, B. Psicologia Social. 22. ed. Rio de Janeiro: Editora Vozes, 2003.

SCHUMACKER, R. E.; LOMAX, R. G. A beginner's guide to structural equation modeling. Lawrence Erlbaum Associates: New Jersey: 2004. 
SCHWARTZ, J.; MILLER, T. The Earth's best friends. American Demographics, 13(2), 26-35, 1991.

SEROA DA MOTTA, R. Social and economic aspects of CDM options in Brazil. In: BARANZINI, A.; BUERGENMEIER, B. (Eds.). Climate change: issues and opportunities for developing countries, special issue. International Journal of Global Environmental Issues, v. 2, n. 3/4, 2002.

SHROUT, P.E.; BOLGER, N. Mediation in experimental and nonexperimental studies: new procedures and recommendations. Psychological Methods, Washington, v.7, n.4, p.422-445, Dec. 2002.

SOLOMON, M. R. O comportamento do consumidor: comprando, possuindo e sendo. Trad. Lene Belon Ribeiro. Porto Alegre: Bookman, 2011.

SOUZA, M. C. G. L.; CASOTTI, L. M.; LEMME, C. F. Consumo Consciente como Determinante da Sustentabilidade Empresarial: Respeitar os Animais pode ser um bom negócio? Rev. Adm. UFSM, Santa Maria, v.6, ed. especial, p. 229246, mai. 2013.

STERN, P. C. Psychology and the Science of Human-Environment Interactions. American Psychologist, v. 55, p. 523-530, 2000.

STERN, P. C.; OSKAMP, S. Managing scarce environmental resources. In: D. Stokols \& I. Altman (Orgs.), Handbook of Environmental Psychology, p. 1043-1088. NY: John Wiley \& Sons, 1987.

TABACHNICK, B. G.; FIDEL, L. S. Using Multivariate Statistics. 4. ed. Needham Heights: Allyn and Bacon, 2001.

TARRANT, M.; CORDELL, K. The effects of respondent characteristics on environmental attitude-behavior correspondence. The Journal of Environmental Education, v. 29, p. 618- 637, 1997.
THURSTONE, L. L. Las actitudes pueden medirse. In: SUMMERS, Gene F. Medición de actitudes. México: Trillas, 1976.

VAN LIERE, K. D.; DUNLAP, K.; NOE, F. P. Outdoor Recreation and Environmental Attitudes: Further Examination of the Dunlap-Hefferen Thesis. Rural Sociology, v. 46, p. 505-513, 1981.

VELTER, A. N.; BATTISTELLA, L. F.; GROHMANN, M. Z.; CASTRO, A. E.; COSTA, V. F.; HERMANN, R. E. Atitudes dos Consumidor a Partir da Teoria das Pistas e da Consciência Ambiental: Contribuições ao Estudo do Green Marketing. Rev. Adm. UFSM, Santa Maria, v. 2., n. 3, p. 399-416, set./dez. 2009.

WAHID, N. A.; RAHBAR, E.; SHYAN, T. S. Factors Influencing the Green Purchase Behavior of Penang Environmental Volunteers. International Business Management, 5, p. 3849, 2011.

WEBSTER JR., F.E. Determining the characteristics of the socially conscious consumer. Journal of Consumer Research, v. 2, n. 3, p. 188-196, 1975.

WHITLAR, D.B.; GEURTS, M.D.; SWENSON, M.J. New product forecasting with a purchase intention survey. The Journal of Business Forecasting Methods Systems, v. 12, n. 3, p. 1-18, 1993.

WILD, H. A Study of Awareness, Understanding and Usage of Environmental Claims: Summary of Main Findings. London: National Consumer Council, 1995. 